\title{
SIMULATION OF PERSONNEL IN ARFORGEN TO PREDICT EFFECTS OF STRUCTURE, POLICY, AND DEMAND CHANGES
}

\author{
David W. Hughes \\ Paul D. Kucik \\ Operations Research Center of Excellence \\ Department of Systems Engineering \\ West Point, NY 10996, USA
}

\author{
Mark M. Zais \\ Strength Analysis and Forecasting Division \\ Army G1 \\ Washington, DC 20310, USA
}

\begin{abstract}
The restructuring of the U.S. Army's active component to 45 Brigade Combat Teams and 13 Combat Aviation Brigades, along with the adoption of the Army Force Generation process, have fundamentally changed Army force structure across rank and specialty while also transforming the model and cycle by which units are manned. In order to meet manning requirements for the planned force structure in support of potential conflicts worldwide, the Army must reassess the manning processes and policies used to achieve these goals. This research utilizes a discrete event simulation model of individual Soldiers as they progress through an Army career, including the demands for soldiers in theater. Simulation results are generated for multiple critical specialties and analyzed to determine the effect of deployment policies on the individual soldier. The Vice Chief of Staff of the Army (VCSA) has designated this effort as a priority modeling effort.
\end{abstract}

\section{INTRODUCTION}

The Army refers to the time a Soldier spends deployed overseas in a combat environment as Boots on the Ground time, or BOG. Conversely, the time a Soldier spends between deployments is known as "dwell." This BOG:Dwell ratio is an important, highly visible statistic, that serves as a leading indicator of recruiting, retention, and morale issues for the Army, its Soldiers, and their families.

The restructuring of the active component to 45 Brigade Combat Teams (BCTs) and 13 Combat Aviation Brigades (CABs), along with the adoption of the Army Force Generation (ARFORGEN) process, have fundamentally changed Army force structure across rank and specialty while also transforming the model and cycle by which units are manned. In addition, the Global War on Terror (GWOT) has increased the importance of manning units to full strength while also placing significant burdens on Soldiers and families. In order to meet personnel requirements for the planned force structure in support of potential commitments worldwide, the Army G1 must frequently reassess the manning processes and policies used to achieve these goals. Thorough assessment of these policies has required modeling of the processes across the lifecycles of both units and individual Soldiers.

In 2009, the VCSA designated BOG:Dwell analysis as a priority modeling effort because he recognized how much stress was being placed on the force and wanted to know which type of Soldiers were the most stressed. He heard stories of young Soldiers who spent nearly $50 \%$ of their time in service deployed in support of on-going operations. So impacted by these stories, he wanted to know if there was anything he could do (such as further restructuring the Army or changing policy) that would have positive effects on the Army and its Soldiers. 


\section{BACKGROUND}

In September 2008, Matt Dabkowski (from West Point's Operations Research Center of Excellence (ORCEN)) and Mark Zais (from the Army G1 Strength Analysis and Forecasting Division) started this project, initially focusing on what was considered the Career Management Field (CMF) with the highest density in BCTs, which was the 11 Series (Infantry). The Army wanted to meet its stated goal of giving Soldiers two years of dwell following a one year deployment. Stated another way, the Army's BOG:Dwell goal was 1:2. In the early 2000s, it was common for key leaders and decision makers to look at unit BOG:Dwell ratios to see if they were meeting their stated goal. At the request of the Army's Human Resources Leadership, Dabkowski and Zais completed research, hereafter referred to as "previous ORCEN/G1 research", building a model (using ProModel (C) simulation software) to test the appropriateness of using unit BOG:Dwell ratios as a proxy for individual Soldier ratios. Their results are found in their technical report titled "Analysis of Unit and Individual BOG:Dwell in Steady-State ARFORGEN" dated 16 July 2009. Today's analysis of the effect of the Army Force Generation Process on the individual Soldier's BOG:Dwell ratio, draws from the initial model developed by the ORCEN and the Army G1. Recent efforts have been focused on improving the model so that it is more scalable, streamlined, and efficient. Ultimately, the desired end state is a simulation that is capable of both estimating the individual dwell statistics by grade for many critical Military Occupational Specialties (MOSs) and producing other residual unit manning and individual attribute statistics.

\section{UNIT BOG:DWELL vs. INDIVIDUAL BOG:DWELL}

Previous ORCEN/G1 research found that unit dwell is not a sufficient proxy for individual Soldier dwell (Dabkowski et al., 2009). This is due to the fact that individual Soldiers do not stay with the same unit throughout their careers. Most change duty stations every 1-3 years whereas most units (certainly not all) were scheduled to deploy once every three years. So, for a unit deploying once in three years, the unit BOG:Dwell will be 1:2. But if a Soldier, coming off a one year dwell since their last deployment, gets stationed at a unit that just had two years of dwell and that is just about to begin a deployment, then that individual Soldier will have a BOG:Dwell of 1:1. Thus, unit dwell can be significantly longer than many of the Soldiers' individual dwell times.

The model calculates unit dwell times based on output from Forces Command's (FORSCOM's) ARFORGEN Synchronization Tool (AST), which is a ProModel based discrete event simulation used by FORSCOM to determine the best sequencing of units into Iraq and Afghanistan, given the available information.

Previous ORCEN/G1 research also found that for the 11 Series, grade is a significant factor in individual Soldier dwell time (Dabkowski et al. 2009). Junior enlisted Soldiers often had worse BOG:Dwell statistics than more senior enlisted infantry Soldiers. They also convinced senior leadership that the median, instead of the average, was the most appropriate measurement of central tendency for the BOG:Dwell ratio (Dabkowski et al. 2009). This is due to the fact that outliers heavily skew averages and dwell statistics do not produce a symmetrical distribution.

Based upon the significance of these findings, the VCSA requested that this analysis be expanded to include other critical specialties, including the remaining BCT centric CMFs (13 - Field Artillery \& 19 Armor), the CAB centric MOSs (all Aviation MOSs in CMF 15), and selected critical enabler MOSs.

\section{FUNCTIONAL DECOMPOSITION}

We generated a simulated Army, assigned and deployed its Soldiers, and replicated their career progression in a manner similar to the previous ORCEN/G1 research. Thus, the functional decomposition found below is virtually unchanged (Dabkowski et al. 2009). Figure 1 is called an Integration Definition for Function Modeling Diagram (IDEF0). 


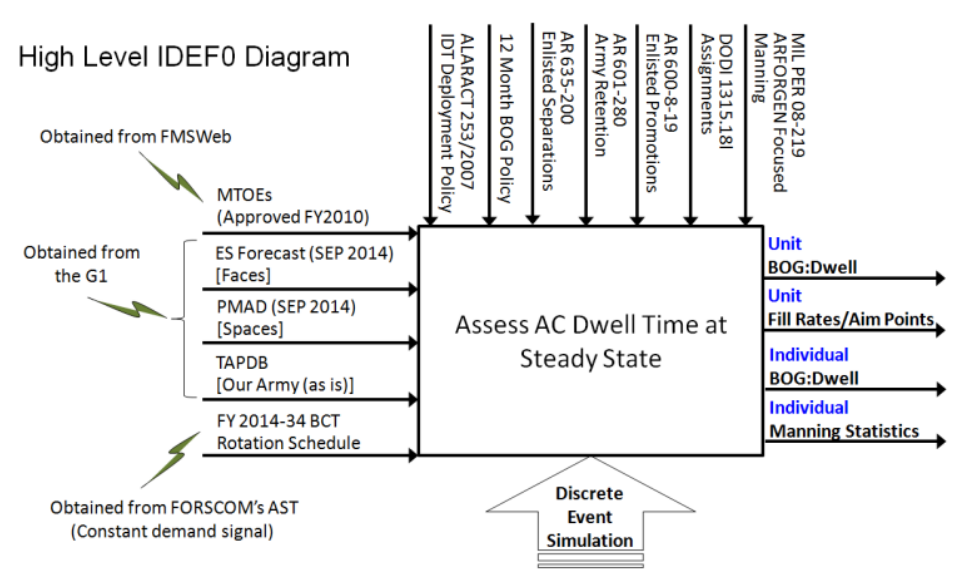

Figure 1: IDEF0 - Assessing Individual BOG:Dwell

\subsection{Inputs}

Represented by the arrows on the left side of Figure 1, the inputs to our model are as follows:

MTOEs: We modeled the entire Army, building structure for BCTs, CABs, Headquarters units, TDA units, numerous supporting units, and an "Other" category to accommodate operational units that are not incorporated in the inputted ARFORGEN Synchronization deployment schedule. Requirements are disaggregated by grade, MOS, and location (Continental United States (CONUS), Outside the Continental United States (OCONUS), or Korea). Table 1 lists types of units and quantities for the BCTs, the CABS, the Headquarters units, and 20 enabling units.

Table 1: Quantities of units by type.

\begin{tabular}{|l|r||l|r|}
\hline \multicolumn{2}{|c|}{ QTY OF UNITS BY TYPE } & \multicolumn{2}{c|}{ QTY OF UNITS BY TYPE } \\
\hline Type & Qty & Type & Qty \\
\hline BCT HBCT & 17 & ADA BATTERY & 6 \\
\hline BCT IBCT & 20 & ADA BN & 9 \\
\hline BCT SBCT & 8 & MAMD BN & 2 \\
\hline CAB Light & 2 & HHB ADA BDE & 6 \\
\hline CAB Medium & 6 & SPACE DET & 3 \\
\hline CAB Heavy & 5 & HHC MI BDE & 5 \\
\hline HdQtrs CONUS & 8 & HHC MP BDE & 5 \\
\hline HdQtrs OCONUS & 1 & HIN MGMT CTR & 6 \\
\hline CHEM BDE & 1 & HR SUST CTR & 4 \\
\hline CHEM BN & 3 & H CHECAP & 6 \\
\hline ACQ CONTR BN & 7 & FIRE SIGN BN & 12 \\
\hline EOD CO & HHC BNE & 7 \\
\hline PUB AFF DET & 14 & HHC MED BDE & 6 \\
\hline
\end{tabular}

Each of these units has unique requirements for Soldiers by MOS and grade. To determine these manning requirements, we pulled the most recently approved FY2011 MTOEs for each type of unit using the Human Resources Command's (HRC's) web-enabled application known as Force Management System Website (FMSWeb).

Enlisted Strength Forecast (Faces): We estimated the Army's Operating Strength (OS), or faces, using the same method employed in previous ORCEN/G1 research (Dabkowski et al. 2009). This forecasted strength by grade and MOS is generated by another Army G1 model called the Active Army Strength Forecaster (A2SF). 


\begin{tabular}{|r|r|r|r|c|c|c|c|r|}
\hline 19,384 & Col Ref & 4 & 5 & 6 & 7 & 8 & 9 & 10 \\
\hline INDEX & MOS & E3 & E4 & \multicolumn{1}{|c|}{ E5 } & E6 & E7 & E8 & E9 \\
\hline 95 & $35 \mathrm{~F}$ & 1160 & 1160 & 1797 & 1362 & 749 & & \\
\hline 96 & $35 \mathrm{G}$ & 258 & 393 & 313 & 296 & 150 & & \\
\hline 97 & $35 \mathrm{~L}$ & & & 418 & 328 & 328 & & \\
\hline 98 & $35 \mathrm{M}$ & 447 & 901 & 750 & 744 & 315 & & \\
\hline 99 & $35 \mathrm{~N}$ & 601 & 600 & 734 & 537 & 350 & & \\
\hline 100 & $35 \mathrm{P}$ & 286 & 329 & 585 & 566 & 380 & & \\
\hline 101 & $35 \mathrm{~S}$ & 149 & 293 & 304 & 238 & 116 & & \\
\hline 102 & $35 \mathrm{~T}$ & 3 & 305 & 131 & 167 & 91 & 26 & 13 \\
\hline 103 & $35 \mathrm{X}$ & & & & & & 223 & 76 \\
\hline 104 & $35 \mathrm{Y}$ & & & & & & 96 & 15 \\
\hline 105 & $35 \mathrm{~V}$ & & & & & & 249 & 52 \\
\hline
\end{tabular}

Figure 2: Enlisted Strength Forecast for the 35 Series

Figure 2 presents an example of disaggregated requirements by grade for MOSs within CMF 35 . In this case, the Army will have 19,384 Soldiers from the 35 Series available for assignment in September 2014.

Personnel Management Authorization Document (Spaces): Similar to the faces above, the Force Structure Allowance (FSA), or spaces, is the authorized positions for Soldiers by MOS and grade. Given the Personnel Management Authorization Document (PMAD), the FSA represents the approved structure for the Active Component. This is the structure that must be filled by the Army's OS.

Total Army Personnel Databases (Attributes): To model the characteristics, or attributes, of the Soldiers, we sampled from the Total Army Personnel Database (TAPDB) with replacement (Dabkowski et al. 2009). Therefore, each Soldier that is created is randomly assigned a set of attributes (Time on Station, Time in Grade, Contract Type, Month to ETS, and Time in Service) from samples of historical data from a selected period of time. The importance of applying samples of historical attribute data to simulation entities during initiation is that it prevents us from having to mature the attributes of the initial Soldiers and allows us to bypass a lengthy warm-up period at the beginning of the simulation.

Rotation Schedule: FORSCOM provides an AST schedule which allows us to simulate ARFORGEN deployments. The output from FORSCOM's AST (small portion shown in Figure 3) provides us with the critical Latest Arrival Date (LAD) and Return (R) date for each rotating unit during our 20-year analysis period. From the LAD and R, we are able to determine all other dates, like LAD-90 and R+90.

\begin{tabular}{|c|c|c|c|c|c|c|}
\hline \multicolumn{7}{|c|}{ RAW DATA - OUTPUT FROM AST - Updated MAR 01, 2011} \\
\hline WDJVFF & $1 \mathrm{IDCAB}$ & $A C$ & SourcingDecision & 365 & $3 / 2 / 2032$ & 3/1/2033 FY32 \\
\hline WDJVFF & 1 ID CAB & $A C$ & BOGMetric & 365 & $3 / 2 / 2032$ & 3/1/2033 FY32 \\
\hline WDJVFF & 1 ID CAB & $A C$ & DwellMetric & 365 & $3 / 2 / 2033$ & 3/1/2034 FY33 \\
\hline WDJVFF & 1 ID CAB & $A C$ & Available & 365 & $3 / 2 / 2034$ & 3/1/2035 FY34 \\
\hline WDJVFF & 1 ID CAB & $A C$ & DwellMetric & 365 & $3 / 2 / 2035$ & 2/29/2036 FY35 \\
\hline WDJVFF & 1 ID CAB & AC & Available & 365 & $3 / 1 / 2036$ & 2/28/2037 FY36 \\
\hline WDJVFF & 1 ID CAB & $\mathrm{AC}$ & DwellMetric & 365 & $3 / 1 / 2037$ & 2/28/2038 FY37 \\
\hline WDJVFF & 1 ID CAB & $A C$ & Available & 365 & $3 / 1 / 2038$ & 2/28/2039 FY38 \\
\hline WDQWAA & 0101 HR COMPANY RECAP & $A C$ & DwellMetric & 365 & $3 / 16 / 2012$ & 3/15/2013 FY12 \\
\hline WDQWAA & 0101 HR COMPANY RECAP & $\mathrm{AC}$ & SourcingDecision & 365 & $3 / 16 / 2013$ & 3/15/2014 FY13 \\
\hline WDQWAA & 0101 HR COMPANY RECAP & $\mathrm{AC}$ & BOGMetric & 365 & $3 / 16 / 2013$ & 3/15/2014 FY13 \\
\hline WDQWAA & $0101 \mathrm{HR}$ COMPANY RECAP & AC & DwellMetric & 652 & $3 / 16 / 2014$ & 12/27/2015 FY14 \\
\hline WDQWAA & 0101 HR COMPANY RECAP & $A C$ & SourcingDecision & 365 & $12 / 28 / 2015$ & 12/26/2016 FY16 \\
\hline
\end{tabular}

Figure 3: Small Portion of FORSCOM's ARFORGEN Synchronization Tool

While previous ORCEN/G1 research noted that the AST had "several shortcomings [that] prevented it from serving as a plug-and-play input" (Dabkowski et al. 2009), we set out to eliminate these shortcomings. With programming assistance from ProModel (C), we were able to code the model so that we can now cut and paste the schedule from FORSCOM AST output directly into our model without any reformatting required. A macro 'Create Schedule' button generates the input formatted for simulation.

One example of a recent steady-state rotation scenario had the following parameters:

- $\quad$ Start Date: October 1, 2014 
- $\quad$ End Date: September 30, 2034

- Deployment Length: 365 days

- RIP-TOA Overlap: 25 days

- MRE Date: LAD-90

- Active Component Demand: 1 Corps/3 Divisions/15 BCTs/41K Enablers

Table 2: Shows the Active Component demand in our scenario.

\begin{tabular}{|l|c|}
\hline Units & Demand Per Year \\
\hline Acquisition Contracting BN & 2 \\
\hline ADA Battalion (Patriot/MEADS) & 1 \\
\hline ADA Battery (THAAD) & 1 \\
\hline Chemical BDE & 1 \\
\hline Chemical BN & 1 \\
\hline Combat AVN BDE (CAB) & 4 \\
\hline CORPS HQ & 1 \\
\hline Division HQ & 3 \\
\hline EOD BN & 3 \\
\hline Financial Management CTR & 1 \\
\hline HHB ADA BDE (EAC) & 1 \\
\hline HHB FIRES BDE & 3 \\
\hline HHC EN BDE & 2 \\
\hline
\end{tabular}

\begin{tabular}{|l|c|}
\hline Units & Demand Per Year \\
\hline HHC Expeditionary Signal BN & 4 \\
\hline HHC Medical Brigade & 1 \\
\hline HHC MI BDE (MIB & 1 \\
\hline HHC MP BDE & 1 \\
\hline HR CO RECAP & 2 \\
\hline Human Resources Sustainment Center & 1 \\
\hline HBCT & 6 \\
\hline IBCT & 8 \\
\hline MAMD BN (Avenger/SLAMRAAM) & 1 \\
\hline Public Affairs DET & 7 \\
\hline Space Detachment & 1 \\
\hline SBCT & 1 \\
\hline
\end{tabular}

\subsection{Controls}

Represented by the arrows entering the top side of the IDEF0 diagram in Figure 1, the controls in this model govern how Soldiers mature (separation, reenlistment, promotion, and assignment), how units are manned, and human resource policies that may affect the degree to which the Army can stress the force. Several policies and regulations govern these processes, including ALARACT 253/2007 IDT Deployment Policy, 12 Month BOG Policy, AR 635-200 Enlisted Separations, AR 601-280 Army Retention, DODI 1315.18p - Procedures for Military Personnel Assignments, and ARFORGEN Focused Manning (AFM). Each of these documents is included in the references.

\subsection{Mechanism}

In order to accept the input, implement controls, and generate output, we decided to use discrete event simulation, a technique which "accounts for interdependencies and variation...provid[ing] insights into the complex dynamics of a system that cannot be obtained using other analysis techniques" (Harrell et al., 2004). With this in mind, we elected to use the discrete event simulation software package ProModel (C). It is worth pointing out that Promodel is used in FORSCOM's AST model and that helps to facilitate interoperability (Dabkowski et al. 2009).

\subsection{Output}

The BOG:Dwell ratio that our model produces is an aggregated measure that indicates the deployment stress on the Army's Soldiers. Since we want to be consistent with the Army's IDT policy, we want to capture the time a Soldier spends at home between deployments. With this in mind, after dividing each deploying Soldier's dwell by his/her BOG, we could take the mean of the sample, leaving us with a single, representative metric for the whole. However, because this distribution is significantly skewed to the left we reported the median, as was done in previous ORCEN/G1 research (Dabkowski et al. 2009). Our model still computes averages so that we can compare the median and the average. Finally, we also decided to represent the individual dwell statistics as a histogram and box plot. Specifically, we were able to capture the distributions of individual BOG:Dwell ratios by CMF alone and by MOS and grade. This allowed us to report a rich set of statistics that fully described the deployment stress on the force. 


\section{SIMULATION STRUCTURE}

The ProModel simulation developed through previous ORCEN/G1 research was "nearly 5 megabytes in size, consist[ed] of 12 entity types, 47 arrays, 121 attributes, 280 variables, 295 macros, 1020 locations, 1300 processes, and 32 subroutines" (Dabkowski et al., 2009). This model was only for the 11 Series, and it took over 27 hours to run. One of our goals for 2011 model was to speed up the run time while adding the ability to analyze multiple, critical MOSs. With contracted programming support from ProModel, the simulation run time has been significantly reduced. The largest CMF (11 Series) can be simulated in less than 2 hours, which is $7 \%$ of the original run-time or a $1500 \%$ increase in speed.

Three macro-enabled Excel input files provide the input for this discrete event simulation: (1) Data File Input - v9.xlsm, (2) Schedule File Input - v3.xlsm, and (3) 2010 Inventory (5-31-10).xlsm.

\subsection{Data File Input - v9.xlsm}

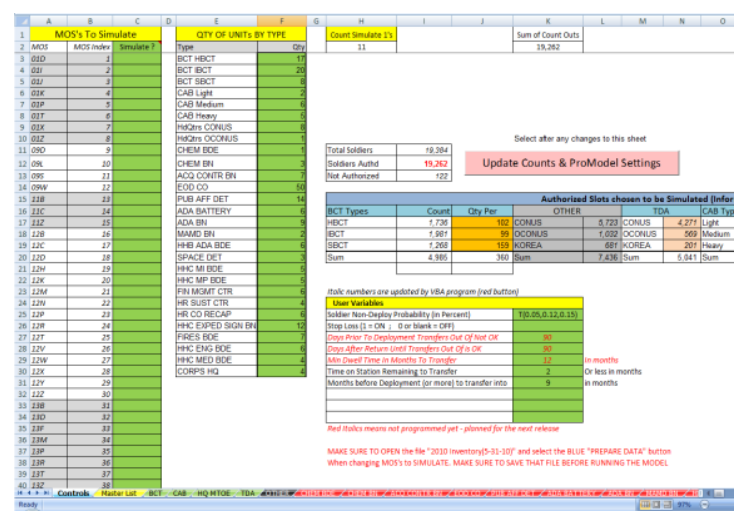

Figure 4: Screen shot of the 'Controls' tab in our 'Data File Input - v9.xlsm' file

Figure 4 is a screen shot of 'Data File Input - v9.xlsm', which provides a readily accessible way to track and modify the model parameters used to represent Army structure and some aspects of policy. This input data is contained in the 30+ tabs (labeled Master List, BCT, CAB, HQ MTOE, TDA, OTHER, Structure for 20 Enabler Units, Inventory Forecast 2014, Promotion Eligibility, Initial Contracts, and Reenlistment Contracts).

\subsection{Schedule File Input - v3.xlsm}

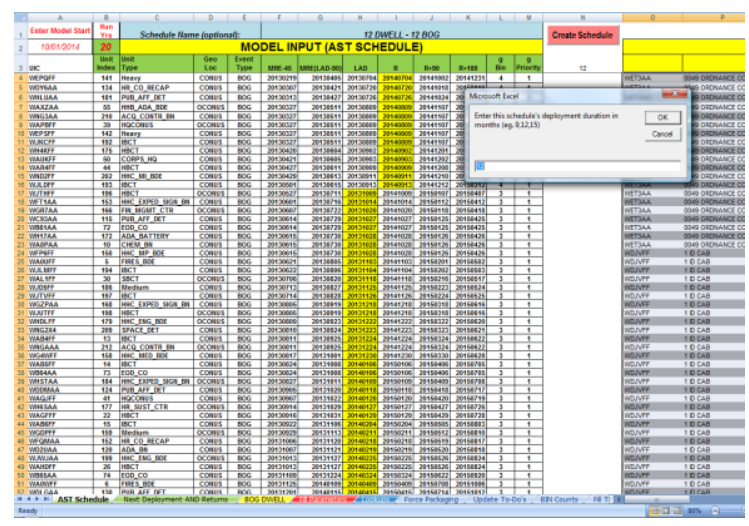

Figure 5: Screen shot of the 'AST Schedule' tab in our 'Schedule File Input - v3.xlsm' file 
Across the bottom of this screen shot in Figure 5, there are 9 more tabs, but the only important one is the 'Fill Parameters' tab. Clicking on that red 'Fill Parameters' tab, allows the user to change the fill target percentages before, re-running the model.

\subsection{Inventory (5-31-10).xlsm}

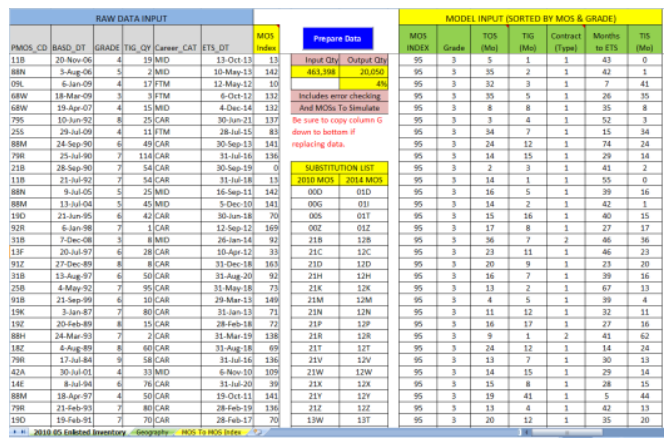

Figure 6: Screen shot of the '2010 05 Enlisted Inventory’ tab in our '2010 Inventory (5-31-10).xlsm' file

The '2010 Inventory (5-31-10).xlsm' Excel file, depicted in Figure 6, provides a logical and transparent method for generating the population of Soldiers in the simulation based on the current inventory of Soldiers. The Excel interface facilitates model updates.

\subsection{BOG Dwell - New v58.mod (ProModel File)}

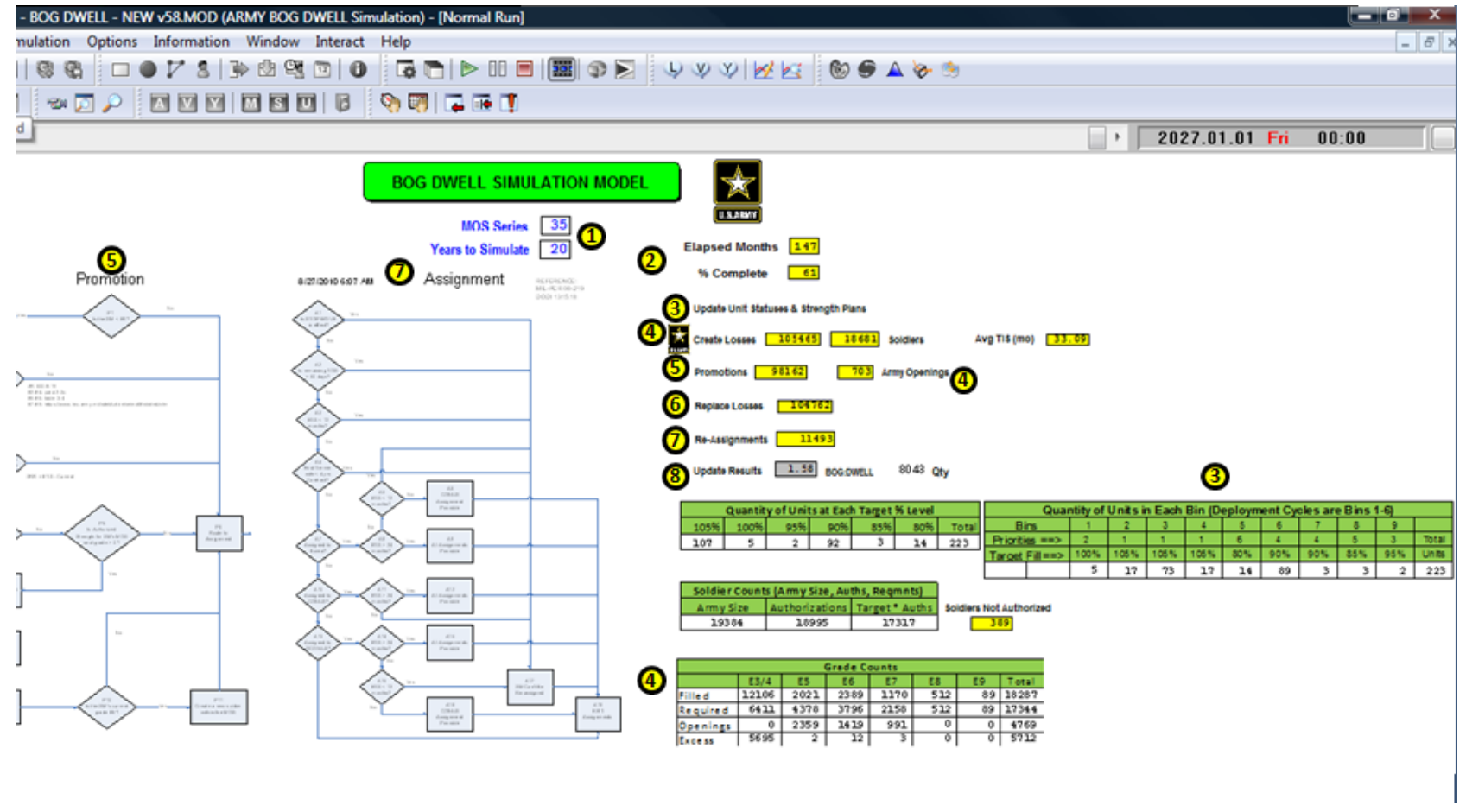

Figure 7: Screen shot of the animation page of our simulation that is built in ProModel

Once the three Excel input files have been open, updated, and saved, we can now move to the ProModel interface. Figure 7 depicts a screen shot of the animation page. To walk through the simulation structure, we have numbers on the figure above and will discuss each number in order. 


\section{Hughes, Zais, Kucik}

1. Depicts the MOS Series (or CMF) being simulated and the length of the simulation run (in years).

2. Continuously updates the months that have elapsed during the simulation run and lets the user know the percentage of the run that is complete.

3. The table to the far right shows how many units are in each fill priority bin. This is dynamically updated every month. The table right next to it gives the roll up of how many units are filled to $105 \%, 100 \%, 95 \%, 90 \%, 85 \%$, and $80 \%$.

4. Every month, Soldiers are "lost" from the simulation for a variety of unforeseen administrative or punitive reasons. Every loss creates an opening in the model. For example in the screen shot above, 703 losses occurred in month 147, bringing the total number of 35 Series Soldiers down from 19,384 to 18,681. A table at the bottom of the animation page serves as a checks and balances to see if each grade is getting what they require. It is easy to see that there are excess Soldiers in the Army in the grades of E3/E4, but there are numerous openings for E5, E6, and E7.

5. Now that there are openings, or spaces in the PMAD, that need to be filled, we first fill those openings by running each and every Soldier through the promotion logic. If a Soldier was eligible for promotion, but could not move because there was no opening for their next grade, then we did not promote them. Once there is an opening as a result of a loss (step 4), then we first look to promote within the same unit, then within the same geographic location, and then finally to other geographic locations (i.e. the Soldier is PCS'd given he/she meets the requirement for PCS). The bottom line is that we are promoting to requirements, which is how the Army G1 manages promotions.

6. Any openings that are not filled as a result of the promotion logic causes new E3s to be created to get the total number of 35 Series Soldiers back to 19,384. The number of Soldiers that are replaced (created) in month 147 are added to the cumulative 'Replace Losses' amount and are tracked on screen.

7. Next, every Soldier goes through the assignment logic. If the Soldier meets the criteria for reassignment and there is a hole in the Army at another location for them, then they will PCS. This will cause the 'Re-assignment' amount to increase on the animation screen. If the Soldier's remaining TOS is 2 months or less and there is no hole available in the Army, then they will not PCS and their remaining TOS will decrease by 1 month. However, their remaining TOS will never go below 1 month. As a result, that Soldier will attempt to PCS every month until a hole opens up. The only other way for the 'Re-assignments' counter to increase on screen is when a promotion occurs that moves a Soldier from one duty location to another.

8. Finally, our model is constantly updating the BOG:Dwell ratio. The screenshot shows the average dwell for the 35 Series (at month 147) as 1.58 years after 8,043 data points have been recorded.

\section{CAPABILITIES}

First and foremost, this model is scalable. Unlike the original version of this model, it is capable of modeling any MOS that is included in FORSCOM's AST. Currently, the model can run the BCT centric CMFs of 11, 13, and 19. Additionally, it can run the Combat Aviation Brigade (CAB) centric CMF, which is 15 . Finally, it is able to model five critical enabler CMFs $(12,25,31,35$, and 89) whose units are included in the AST schedule.

How else is this model significantly improved from the version released in 2009? The data input and update process has been significantly streamlined. First, instead of having decision logic in our model (for example, will the Soldier reenlist or not? Will the Soldier retire or not? Will the Soldier get in trouble and get separated or not?), we aggregated all the loss rates by MOS. This significantly sped up our model. Second, we no longer have to reformat FORSCOM's AST. We can simply cut and paste it directly into our Excel input file called 'Schedule File Input - v3.xlsm.' Lastly, we got rid of nearly every entity and location within ProModel and instead ran sub-routines, which are so much faster. This streamlining of data greatly reduced our simulation run-times. The 11 Series (our largest CMF) used to 
take 27 hours to run. Now it only takes 1 hour and 45 minutes to run. Some smaller CMFs only take about 30-40 minutes to complete a run.

\section{RESULTS}

We ran a steady-state simulation for the following Career Management Fields (CMFs): 11 (Infantry), 12 (Engineers), 13 (Field Artillery), 15 (Aviation), 19 (Armor), 25 (Signal), 31 (Military Police), 35 (Military Intelligence), and 89 (Explosive Ordnance Disposal). We previously defined the steady-state as a 20-year analysis period starting on October 1, 2014 where (a) the AC Demand scenario is 1 Corps, 3 Divisions, 15 BCTs, 41K Enablers (normally represented as: 1/3/15/41K), (b) the standard deployment length is 1 year, (c) the RIP-TOA overlap is 25 days, and (d) the Army's authorized end strength is 463,398 Soldiers. After running the simulation, we analyzed the output data using the statistical software program MiniTab.

The boxplot is a great way to visually convey statistical information. In particular, each boxplot's colored, rectangular region represents the middle $50 \%$ of the sample (or interquartile range); the horizontal line inside of the colored region is the median; and the line or whiskers emanating from its top and bottom represent the sample's upper and lower 25\%. Any values which are greater than 1.5 times the interquartile range are considered outliers, and these unusual observations are plotted as asterisks. We removed these outliers from some of our figures to "clean" them up. (See the difference between Figures $8 \mathrm{a}$ (outliers displayed) and $8 \mathrm{~b}$ (outliers removed). With the explanations out of the way, we can now focus on interpreting the results.

Figure 8a compares dwell years by grade for the 11 (Infantry), 13 (Field Artillery), 15 (Aviation), and 19 (Armor) CMFs. Since all of the colored regions of the boxplots fall below the 2-year dwell line (shown in red), we can conclude that more than $75 \%$ of the Soldiers in these CMFs failed to experience a 1:2 BOG:Dwell ratio. In fact, only the 13 Series E7s and the E7s and E8s in the 19 Series have a significant portion of Soldiers getting 2 years of dwell (their upper whisker crosses over the 2-year dwell line). The lower enlisted Soldiers in the BCTs and CABs are not faring well at all. Specifically, in the BCTs, the dwell year medians for the skill level 1 (SL1) Soldiers (which equates to the E3s and E4s in our model) are all below the E6s, E7s, and E8s.

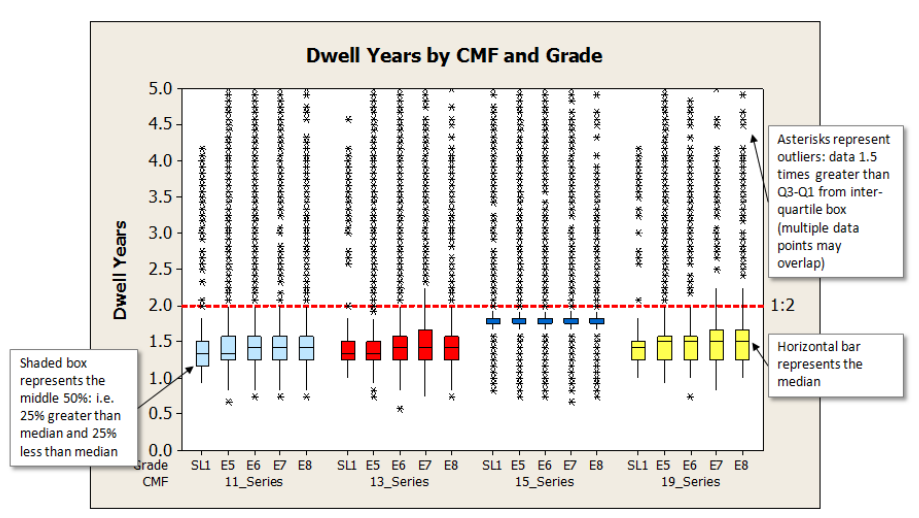

Figure 8a: Dwell Years by CMF and Grade $(11,13,15$, and 19 Series $)$

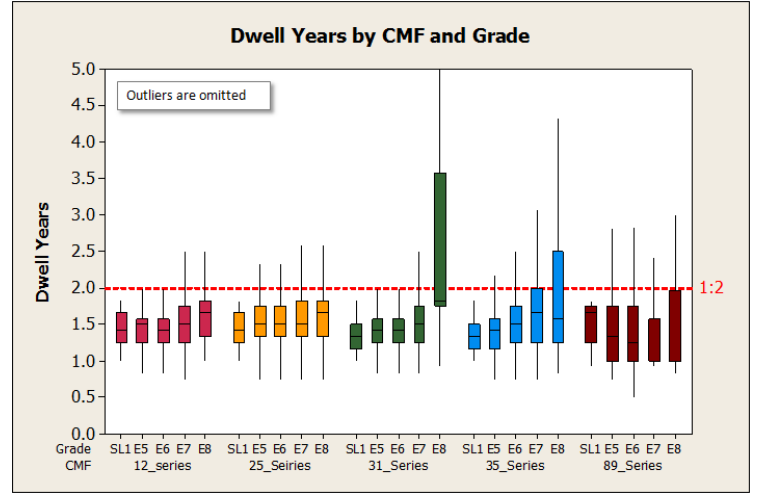

Figure 8b: Dwell Years by CMF and Grade $(12,25,31,35$, and 89 Series $)$

Next, we compared the 12 (Engineers), 25 (Signal), 31 (Military Police), 35 (Military Intelligence), and 89 (Explosive Ordnance Disposal) Series. See Figure 8b. These are five of the most critical enablers CMFs that the Army G1 wanted us to analyze. It is again clear to see that most Soldiers are not getting 2 years of dwell time. In fact, in each of these CMFs, the median for each grade falls below the red line. Also, only the E8s in the 31 and 35 Series have portions of their boxplot well over the 2-year dwell line. 
Additionally, the SL1s have lowest median dwell times in the 12, 25, 31, and 35 Series. Interestingly, the 89 Series SL1 has the highest median dwell time in its series. However, none of its upper whisker crosses the red line. So essentially, almost every SL1 in the 89 Series is failing to get 2 years of dwell time.

We then wanted to plot the median dwell times for each MOS. See Figure 9a. Only three MOSs $(15 \mathrm{~K}, 31 \mathrm{D}$, and $12 \mathrm{P})$ achieve the Army's BOG:Dwell ratio goal of 1:2. 25E and 35X were the next closest MOSs to achieving the Army's goal. The MOSs that fared the worst are 12C, 35Y, and 89D.

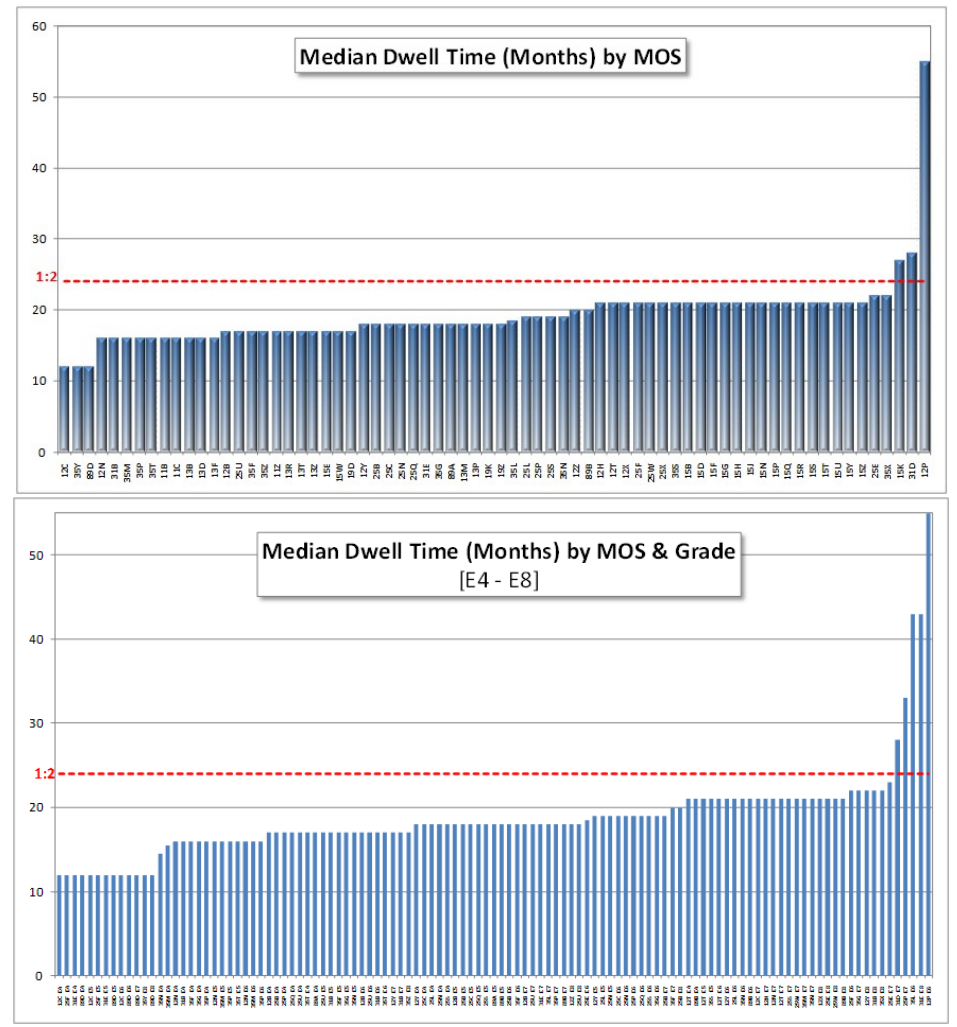

Figure 9a (top) and 9b: Median Dwell Time (in months) by MOS and then by MOS \& Grade (11, 12, 13, $15,19,25,31,35$, and 89 Series)

Next, we graphed the median dwell times for MOS and grade (Figure 9b). (E3s are not depicted because by the time they have a dwell statistic, they have been in the Army long enough to make E4. We also excluded E9s to save space.) Only 15K E7s, 31D E7s, 25P E7s, 35L E6s, 31E E8s, and 12P E6s achieve a median dwell greater than two years. 12C E4s/E5s/E6s, 13M E7s, 13T E7s, 25F E4s/E5s, 31E $\mathrm{E} 4 \mathrm{~s} / \mathrm{E} 5 \mathrm{~s}, 89 \mathrm{D} \mathrm{E} 4 \mathrm{~s} / \mathrm{E} 5 \mathrm{~s} / \mathrm{E} 6 \mathrm{~s} / \mathrm{E} 7 \mathrm{~s} / \mathrm{E} 8 \mathrm{~s}$, and 35Y E8s all tied for the worst median dwell time of 12 months. This makes it clear to see why $12 \mathrm{C}$ and $89 \mathrm{D}$ as a whole fared so poorly.

\section{VERIFICATION AND VALIDATION}

As the famous statistician George Box rightly stated, "All models are wrong, some are useful" (Parnell et al., 2011). As such verification and validation are the methods by which we try to ensure the model is as right as necessary. With this in mind, the following sections briefly address the techniques we used to ensure the correctness and quality of our simulation (Dabkowski et al 2009). 


\section{Hughes, Zais, Kucik}

\subsection{Verification}

Verification essentially answers the question, "Did we build the model right, or does our model operate the way we envisioned it?" While there are many ways to verify a model, we focused on using three techniques, namely iteration, animation, and numerical / graphical observation (Dabkowski et al, 2009).

Iteration: The model's current version is 58. This is a result of repeated testing. Each time a new version is updated, testing is conducted by running the simulation for each of the nine CMFs and analyzing the results.

Animation: While the use of graphics is aesthetically pleasing and useful for conveying a simulation's logic to decision makers, it also facilitates verification (Dabkowski et al, 2009). Our on-screen counters show the model is progressing through time, and we expect to see the numbers constantly update throughout the simulation run. There were a number of errors that were identified by watching the onscreen counters. If we did not have this animation enabled, we would have to wait until the end of the simulation run to start verification. Thus, the animation allowed us to immediately identify anomalies or inconsistencies with historical Army behavior by presenting pertinent information that can be assessed while the model was running.

Numerical and Graphical Observation: As a final check, we compared many numerical results to their required or expected values both throughout and at the end of the simulation runs. For instance, we checked to ensure that the number of Soldiers at the end of a run (by MOS and grade) roughly matched the number at the beginning, and that the contents of each unit closely mirrored its MTOE (Dabkowski et al 2009).

That said, a picture is often worth a thousand debugging statements. As an example, we might want to ensure that the deploying units experience fill percentages that are consistent with AC Manning Guidance during their ARFORGEN manning cycles. One of the figures (that we could not include due to space restrictions) verifies this nicely, as the BCT's actual fill percentages depicted over the time period shown hover between $80 \%$ and $105 \%$. In sum, we are confident that we built the model right (Dabkowski et al 2009).

\subsection{Validation}

Similar to verification, validation answers a question, namely, "Did we build the right model, or does our model mimic the real system closely enough for us to have confidence in its results?" Almost without exception, validating a model is much more difficult than verifying it. Nonetheless, we used the following techniques to establish the validity of our model: extensive use of primary source data, entity trace, and model comparison (Dabkowski et al. 2009).

Primary Source Data: From securing the AST from FORSCOM to using validated strength forecasts from PRS, we used and implemented primary source data whenever possible. While some might question this as a formal validation technique, many have advocated this approach in order to guarantee that the model has high face validity (Sargent 1998). As an example, by resampling our (TIS, TIG, enlistment status) triples with replacement from the TAPDB, we maintained the underlying, statistical dependence of these parameters (Dabkowski et al. 2009).

Entity Trace: As a technique, entity trace essentially records the actions of an entity during a simulation run, so they can subsequently be compared to actual behaviors (Harrell et al., 2004). With this in mind, we performed both partial and complete entity traces throughout the model development (Dabkowski et al. 2009). Figure 10 shows output produced from the model that depicts the career progression of Soldier ID\# 72662. With this output we can chronologically trace the time that this Soldier spent in individual 
units, following him through multiple deployment cycles in order to verify the reasonableness of results at the individual level.

\begin{tabular}{|c|c|c|c|c|c|c|c|c|c|}
\hline 4 & A & & B & C & D & E & $\mathrm{F}$ & G & H \\
\hline 1 & 1 & & 2 & 3 & 4 & 5 & 6 & 7 & 8 \\
\hline 2 & MOS & - & Grade - & Unit $v$ & Unit Type - & Sim Mo. . & BOG Months & Dwell Months - & Soldier ID $\nabla$ \\
\hline 8941 & & 13 & 4 & 192 & 2 & 42 & 12 & 16 & 72662 \\
\hline 27237 & & 13 & 4 & 192 & 2 & 69 & 12 & 15 & 72662 \\
\hline 74469 & & 13 & 5 & 20 & 3 & 135 & 12 & 54 & 72662 \\
\hline 98231 & & 13 & 6 & 30 & 3 & 168 & 12 & 21 & 72662 \\
\hline
\end{tabular}

Figure 10: Screen shot of the career progression of Soldier ID\# 72662

Model Comparison: Since previous ORCEN/G1 research included a well-detailed model validation, we used this validation and their 11 Series results to confirm the accuracy of our 11 Series simulation run.

\section{FUTURE WORK}

One of the results of socializing our modeling results and capabilities has been widespread enthusiasm and support for a rigorous analysis of the Army's BOG:Dwell challenges. Predictably, with this awareness came additional requests for support. PRS already would like us to analyzed five more critical CMFs (42 - Administrative, 68 - Medical, 88 - Transportation, 91 - Maintenance, 92 - Supply \& Logistics). Additionally, the $37^{\text {th }}$ Chief of Staff of the Army has asked the Army G1 to look at the effects of shortening deployments to nine months and what impact that would have on BOG:Dwell ratios. Finally, we have been asked to study the effects of different demand scenarios so we can answer questions regarding whether our current force structure can handle higher demand. With just a few inputs (AST schedule, force structure of the Active Component Army, and current policies), there are a myriad of questions we can answer with our model.

\section{CONCLUSION}

The result of this research is a realistic and useable simulation tool that can assist decision makers in analyzing the future effects of current and proposed demand, structure, and policy changes. As the international environment changes, this tool will allow decision makers to design policy which complies with applicable regulations, law, and procedures and to understand the effect of that Army-level policy on the individual Soldier.

\section{REFERENCES}

Dabkowski, Matt, Zais, Mark, Kwinn Jr., Michael J. and Miller, Kent. 2009. Analysis of Unit and Individual BOG:Dwell in Steady-State ARFORGEN. Technical Report. Operations Research Center of Excellence.

Department of Defense. 2005 (12 January). DODI 1315.18p: Procedures for Military Personnel Assignments.

Department of the Army. 2005. AR 635-200: Active Duty Enlisted Administrative Separations.

Department of the Army. 2006. AR 601-280: Army Retention Program.

Department of the Army. 2007 (November). ALARACT 253/2007: Individual Dwell Time (IDT) Deployment Policy.

Department of the Army. 2008a (10 April). Active Army Twelve Month Boots On The Ground (BOG) Policy.

Department of the Army. 2008b. AR 600-8-19: Enlisted Promotions and Reductions.

Department of the Army. 2008c (10 July). HQDA Active Component Manning Guidance for FY 20082010.

Department of the Army. 2011 (14 March). AR 525-29: Army Force Generation. 
Department of the Army. 2008d. (29 August). MILPER Message Number: 08-219 (AHRC-PL-PN) ARFORGEN Focused Manning.

Harrell, Charles, Ghosh, Biman K., \& Bowden Jr., Royce O. 2004. Simulation Using ProModel. 2nd edition. McGraw Hill, New York.

Parnell, Gregory S., Driscoll, Patrick J., \& Henderson, Dale L. (eds). 2011. Decision Making in Systems Engineering and Management. 2nd edition. Wiley Series in Systems Engineering and Management. John Wiley \& Sons, Inc.

Sargent, Robert G. 1998. Verification and Validation of Simulation Models. 